\section{Pneumonia classification and healthcare-associated pneumonia: a new avenue or just a cul-de-sac?}

\author{
Mark Woodhead
}

Peripneumony, peripleumoniacon and pleurisy were all terms used by the ancient Greeks and Romans to refer to illnesses which include the condition we now know as pneumonia. In the early 19th century, Laennec separated 'pleurisy' from pneumonia, and later that century, Rokitansky was probably the first to recognise bronchopneumonia and lobar pneumonia as separate pathological entitities. In 1938, Reimann ${ }^{1}$ introduced the term 'atypical pneumonia' for a group of pneumonias with a slower evolution and later link with mycoplasma aetiology (although subsequent studies suggest that such a clinical distinction is difficult and not useful in routine practice). The late 19th and 20th centuries were those of microbial discovery and the multiplicity of microbial pathogens that commonly cause pneumonia were recognised. It was only later in the 20th century, with alterations in the structure of society and healthcare facilities, that different patterns of microbial aetiology linked to social and population factors started to be detected, particularly in the USA. A high frequency of pneumonia associated with Gram-negative bacilli was found in a population with a high frequency of alcoholism. ${ }^{2}$ In a US study published in 1973, Gram-negative bacteria were found, perhaps for the first time, to be associated with pneumonia arising in those in institutional residence, ${ }^{3}$ and similar findings were found in patients with pneumonia developing in nursing homes-nursing home-acquired pneumonia (NHAP). ${ }^{4}$ This was the precursor to the current internationally recognised classification of pneumonia by site of origin into either hospital acquired pneumonia (HAP) or community acquired pneumonia (CAP) - a classification that is easy to apply at patient presentation, and which links to a different spectrum of bacterial aetiology, and hence, empirical antibiotic treatment (a classification that unfortunately is not yet recognised in the WHO International Classification of Disease!).

Correspondence to Professor Mark Woodhead, Respiratory Medicine, Manchester Royal Infirmary, Oxford Road, Manchester M13 9WL, UK; mark.woodhead@cmft.nhs.uk
The most recent development in pneumonia classification has been the introduction of the concept of Health Care-associated Pneumonia (HCAP) as described for the first time in the 2005 ATS/IDSA Guidelines. ${ }^{5}$ A growing population residing in long-term care facilities, and an increase in the use of antibiotics and invasive procedures in such settings had created an environment more akin to the hospital than the community. HCAP included four patient subgroups:

- hospitalised in an acute care hospital for two or more days within 90 days of the infection

- resided in a nursing home or long-term care facility

- received recent intravenous antibiotic therapy, chemotherapy, or wound care within the past 30 days of the current infection

- attended a hospital or haemodialysis clinic.

The term, HCAP, was thus expected to predict a higher frequency of Gram-negative and multiresistant bacteria, thus requiring different empirical antibiotic therapy to that used for CAP. Disease classification has evolved with the principle aim of providing a common language to enable epidemiological comparisons and to guide disease management. For such a classification to achieve these objectives mandates that it is grounded on a solid and generalisable evidence base. The validity of HCAP as a clinically useful entity depends on the evidence-base supporting it as a separate condition requiring separate management.

Initial studies from the USA supported this new classification. ${ }^{6}$ However, subsequent studies have introduced doubts, particularly about the worldwide generalisability of the HCAP concept. First of all, studies of NHAP in Europe, unlike those in North America, found a spectrum of microbial causes similar to CAP rather than HAP $^{7-9}$ More recently, studies of HCAP from outside the USA have been published, including the study from Spain, in this journal. ${ }^{10}$ Using prospectively collected data, from a consecutively admitted cohort of adults with pneumonia in 12 Spanish hospitals, a case-control study of 238 adults with CAP and 238 adults with HCAP (definition slightly modified) was performed. A strength of the study is that the frequency of attribution of a microbial cause $(61 \%$ and $58 \%$, respectively) is high compared with other prospective studies of pneumonia aetiology. Unfortunately, in contrast with the attractive premise behind the concept of HCAP, the frequency and spectrum of causative pathogens was similar in HCAP and CAP, with Streptococcus pneumoniae the most common pathogen and pneumonia due to Gram-negative bacteria and antibioticresistant bacteria rare. This then challenges the concept of HCAP as a useful specific entity. How then does this correlate with other studies of HCAP?

An additional single-centre US study ${ }^{11}$ found a much higher frequency of antibiotic-resistant bacteria in HCAP than CAP with Meticillin-resistant Staphylococcus aureus (MRSA) (24.6\% of cases) and Pseudomonas aeruginosa (18.8\%) the predominant organisms. It is notable that $12 \%$ of CAP cases were attributed to MRSA in this study-an unusually high figure compared with most studies. A single-centre study from Korea ${ }^{12}$ and a multicentre study from Japan ${ }^{13}$ also found antibiotic-resistant bacteria to be more common in HCAP. This contrasts with an earlier study from Spain ${ }^{14}$ and one from the $\mathrm{UK}^{15}$ which found no excess of antibiotic-resistant bacteria in patients with HCAP_-findings more similar to the study by Polverino et al in this journal. Perhaps most notable is that, overall, antibiotic-resistant bacteria were very uncommon in these three studies. The only significant aetiological difference was the higher frequency of aspiration pneumonia in HCAP (up to $28.2 \%$ of cases) compared with CAP $(5.5 \%)$ in the study by Garcia-Vidal. The frequency of aspiration pneumonia is variably reported in these studies, in part, due to the difficulty of accurate diagnosis and lack of an agreed definition for this condition. In the study, ${ }^{10}$ conditions likely to increase aspiration risk (neurological disease and dysphagia) were more common in the HCAP cohort.

An additional concern over the HCAP concept is the aggregation of four or more groups of pneumonia patients from different settings in the absence of large studies of aetiology in these groups, other than for the nursing home patients which always numerically dominate any HCAP cohort. Garcia-Vidal et al did try to analyse these subgroups separately, finding similarities and differences within the HCAP heading. A recent study of CAP in haemodialysis patients found good outcomes with narrow-spectrum antibiotic therapy, but did not investigate microbial aetiology. ${ }^{16}$ Despite the above differences, 
all studies appear to agree that outcomes are worse for patients with HCAP compared with CAP. In the current study, a 1 -month mortality in HCAP patients was $12.2 \%$ compared with $4.6 \%$ in CAP. Studies also concur over measures defining the functional capacity or biological fitness of patients as being worse in those with $\mathrm{HCAP}$ - an important determinant of outcome, and perhaps one of the contributors to the increased frequency of aspiration pneumonia in these patients. An interesting finding in the UK study was the higher frequency of application of care limitation orders in those with HCAP which will be at least in part related to this limitation of functional capacity. ${ }^{15}$

So, in conclusion, it appears that the HCAP concept may be a generalisable approach to the identification of pneumonia patients with a worse prognosis. The worse outcome in HCAP is related to patient factors rather than treatment received, and may not, therefore, be amenable to medical intervention, or in other words be preventable. It is uncertain whether using HCAP for its prognostic value adds to the prognostic tools already available for CAP. There appear to be settings in which the HCAP concept can identify a group of patients at higher risk of antibiotic-resistant bacteria, and thus, requiring different empiric antimicrobial therapy compared with others with CAP. This only applies in some healthcare settings, especially those with high underlying bacterial antibiotic resistance rates as found in the USA and some Asian countries. In European centres, the HCAP concept does not appear to identify patients with microbial aetiology different from other CAP. Therefore, in these settings, HCAP should not be used to direct empirical antibiotic therapy. It would have the opposite effect to that intended, and would promote the development of bacterial antibiotic resistance. Further studies of microbial aetiology, treatment and outcome of the HCAP subgroups may be useful, but in the meantime, HCAP may not be a generally useful addition to the recognised pneumonia classification.

\section{Competing interests None.}

Provenance and peer review Commissioned; internally peer reviewed.

To cite Woodhead M. Thorax 2013;68:985-986.

Published Online First 3 September 2013

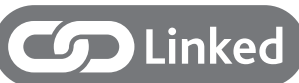

http://dx.doi.org/10.1136/thoraxjnl-2013-203828

Thorax 2013;68:985-986.

doi:10.1136/thoraxjnl-2013-204060

\section{REFERENCES}

1 Reimann H. A An acute infection of the respiratory tract with atypical pneumonia. JAMA 1938;111:2377-84.

2 Sullivan RJ, Dowdle WR, Marine WM, et al. Adult pneumonia in a general hospital. Arch Int Med 1972;129:935-42.

3 Dorf GJ, Rytel MW, Farmer SG, et al. Etiologies and characteristic features of pneumonias in a municipal hospital. Am J Med Sci 1973;266:349-58.

4 Garb JL, Brown RB, Garb JR, et al. Differences in etiology of pneumonias in nursing home and community patients. JAMA 1978;240:2169-72.

5 American Thoracic Society; Infectious Diseases Society of America. Guidelines for the management of adults with hospital-acquired, ventilator-associated, and healthcare-associated pneumonia. Am J Respir Crit Care Med 2005;171:388-416.

6 Kollef MH, Shorr A, Tabak YP, et al. Epidemiology and outcomes of healthcare-associated pneumonia: results from a large US database of culture positive pneumonia. Chest 2005:128:3854-62.

7 Ewig S, Klapdor B, Pletz MW, et al. Nursinghome-acquired pneumonia in Germany: an 8-year prospective multicentre study. Thorax 2012;67: 132-8.

8 Lim WS, Macfarlane JT. A prospective comparison of nursing home acquired pneumonia with community acquired pneumonia. Eur Respir J 2001;18:362-8.

9 Polverino E, Dambrava P, Cilloniz C, et al. Nursing home-acquired pneumonia: a 10-year single-centre experience. Thorax 2010;65:354-9.

10 Polverino E, Torres A, Menendez R, et al. Microbial aetiology of Health Care Associated Pneumonia (HCAP) in Spain: a prospective, multicenter, casecontrol study. Thorax 2013;68:1007-14.

11 Micek ST, Kollef KE, Reichley RM, et al. Health Care-associated pneumonia and community-acquired pneumonia: a single centre experience. Antimicrob Agents Chemother 2007;51:3568-73.

12 Park SC, Kang YA, Park BH, et al. Poor prediction of potentially drug-resistant pathogens using current criteria of health care-associated pneumonia. Respir Med 2012;106:1311-9.

13 Shindo $Y$, Ito $R$, Kobayashi $D$, et al. Risk factors for drug-resistant pathogens in community-acquired and healthcare-associated pneumonia. Am I Respir Crit Care Med Published Online First: 15 July 2013. doi:10.1164/rccm.201301-00790C

14 Garcia-Vidal C, Viasus D, Roset A, et al. Low incidence of multidrug resistant organisms in patients with healthcare-associated pneumonia requiring hospitalization. Clin Microbiol Infect 2011;17:1659-65.

15 Chalmers JD, Taylor JK, Singanayagam A, et al. Epidemiology, antibiotic therapy, and clinical outcomes in health care-associated pneumonia: a UK cohort study. Clin Infect Dis 2011;53:107-13.

16 Taylor SP, Taylor BT. Health care-associated pneumonia in haemodialysis patients: clinical outcomes in patients treated with narrow versus broad spectrum antibiotic therapy. Respirology 2013:18:364-8 